\title{
Current Practice vs. Guideline Based Imaging in Abdominal Radiology in the German Speaking Area: Results of an Online Survey
}

\section{Versorgungsrealität vs. leitliniengerechte Bildgebung in der Abdominalradiologie im deutschsprachigen Raum: Ergebnisse einer Online-Umfrage}

Authors

Affiliations
A. G. Schreyer ${ }^{1}$, J. Wessling ${ }^{2}$, L. Grenacher ${ }^{3}$

Department of Radiology, University Hospital Regensburg, Germany

Department of Radiology, Clemens Hospital, Münster, Germany

Diagnostic Imaging Center, Diagnostik München, Germany
Key words

- abdomen

- CT

- diagnostic radiology

- MR-imaging received 14.7.2015

accepted $\quad 4.12 .2015$

\section{Bibliography}

DOI http://dx.doi.org/

10.1055/s-0041-111846

Published online: 27.1.2016

Fortschr Röntgenstr 2016; 188:

268-279 @ Georg Thieme

Verlag KG Stuttgart · New York . ISSN 1438-9029

\section{Correspondence}

\section{Prof. Andreas G. Schreyer}

Institut für Röntgendiagnostik, Universitätsklinikum

Regensburg

Franz-Josef-Strauß-Allee 11

93051 Regensburg

Germany

Tel.: ++ 49/941/9447401

Fax: ++ 49/941/9447402

andreas.schreyer@klinik.uni-

regensburg.de

\section{Abstract \\ V}

Purpose: The working group for abdominal imaging within the German Roentgen Society (DRG) performed an online survey amongst radiologist concerning the current status of techniques for abdominal imaging. The results of this survey were compared with the most recent guidelines.

Materials and Methods: We performed an online survey open for all members of the DRG during a 76-day period with 65 questions and an overall estimated time for finishing the questionnaire of 15 minutes concerning technical specifications for abdominal radiological imaging. The results were evaluated using descriptive statistics.

Results: 90 of all participants, who filled out the survey covering more than $80 \%$ of the questions, were included in the statistical evaluation. $27 \%$ of all participants were registered radiologists while $73 \%$ represented radiologists working in a hospital. Most participants worked in a managerial position. The participants gave detailed information regarding the radiological techniques used in dedicated organ systems (esophagus, stomach, pancreas, liver, small intestines, colon) regarding acquired contrast phases and oral and intravenous administration of contrast medium.

Conclusion: The results confirm that most radiologists participating in this survey perform their examinations and choose their modalities in conformity with the current existing clinical guidelines. Because most clinical guidelines do not specify radiological examinations in detail, there is a noteworthy heterogeneity of the acquired contrast phases and major divergence in terms of technical parameters. Therefore a joint radiological effort should be initiated for developing and publishing updated radiological parameters for abdominal imaging.

Key Points:

- In German speaking countries indications for abdominal radiological examinations are performed with a high compliance to published guidelines.

- There is a significant heterogeneity regarding the acquisition of different contrast phases for parenchymal abdominal organs.

- There is an urgent need to publish radiological recommendations and guidelines based on current radiological literature for radiological abdominal imaging.

Citation Format:

- Schreyer AG, Wessling J, Grenacher L. Versorgungsrealität vs. leitliniengerechte Bildgebung in der Abdominalradiologie im deutschsprachigen Raum: Ergebnisse einer Online-Umfrage. Fortschr Röntgenstr 2016; 188: $268-279$

\section{Zusammenfassung \\ $\nabla$}

Ziel: In einer Online-Umfrage der Arbeitsgemeinschaft Abdominal- und Gastrointestinaldiagnostik der Deutsche Röntgengesellschaft (DRG) sollte ein aktuelles Abbild der gegenwärtig durchgeführten abdominalradiologischen Untersuchungstechniken in Deutschland erstellt und die Ergebnisse den aktuellen Leitlinien zu diesen Themenbereichen gegenübergestellt und kritisch bewertet werden. Material und Methoden: Den Mitgliedern der DRG, die zu der Umfrage in Newslettern und E-mails eingeladen wurden, stand für 76 Tage eine Online-Umfrage mit insgesamt 65 Fragen und einer Bearbeitungszeit von über 15 Minuten pro Fragebogen zur technischen Durchführung von abdominalradiologischen Fragestellungen offen. Die Ergebnisse der Umfrage wurden mit deskriptiven statistischen Methoden ausgewertet. 
Ergebnisse: Insgesamt beantworteten 90 Teilnehmer die Fragebogen über $80 \%$, die für die Auswertung berücksichtigt werden konnten. Die Umfrage wurde von $27 \%$ niedergelassenen- und $73 \%$ Klinikradiologen durchgeführt, wobei die meisten Teilnehmer in leitenden Positionen waren. Eingeteilt nach Organregionen (Ösophagus, Magen, Pankreas, Leber, Dünndarm, Dickdarm) wurden Angaben zu präferierten akquirierten Kontrastmittelphasen, Arten der oralen und intravenösen Kontrastierung und Auswertemöglichkeiten gemacht.

Schlussfolgerung: Die Ergebnisse der Umfrage zeigen, dass die meisten befragten Radiologen eine leitlinienkonforme Untersuchungstechnik verwenden, wobei die Mehrheit der Leitlinien keinerlei konkrete Angaben zur radiologisch technischen Untersuchungsdurchführung machen. Daher fallen eine beträchtliche Heterogenität der akquirierten Kontrastmittelphasen sowie eine Diversität der möglichen technischen Parameter auf. Es sollte daher angestrebt werden, eine aktuelle evidenzbasierte Empfehlungszusammenstellung für radiologische Untersuchungen zu entwickeln und zu erstellen.

\section{Introduction}

$\nabla$

In recent years, major advances in imaging and therapy have been achieved in the field of abdominal radiology both nationally and internationally $[1,2]$. Systematic clinical studies documented in various radiological publications have sought to optimize existing radiological protocols [3-6]. In addition, new procedures, such as diffusion imaging or functional imaging with molecular methods, have been introduced [6]. In principle, implementing these individual innovations on a broad basis within a short period poses, in the medical world, a problem and challenge that must not be underestimated. To rapidly facilitate efficient state-of-the-art operation in the field of medicine, medical care guidelines have been increasingly implemented around the world. These guidelines are generally compiled for individual bodily systems or diseases by an interdisciplinary committee of experts [7-10]. Guidelines should be systematically developed decisionmaking aids as defined by the Institute of Medicine (IOM) to facilitate correct procedure for specific medical problems. These guidelines are frequently published swiftly so that sound medical care can be achieved on a broad basis according to evidence-based criteria and a review of the most current literature on the particular topic [11, 12]. While representatives from the field of radiology frequently participate in these guidelines, there are barely any radiologically initiated guidelines in Germany that process and systematically evaluate clear, evidence-based recommendations for the correct or optimized execution of radiological examinations. It is thus apparent that diverse guidelines address various radiological examination methods with differing accuracy and in different manners [13-15]. In some cases, only an umbrella term, such as "abdominal" CT", is given as a type of black box term, without providing any detail as to which contrast medium phases should be acquired. In general, the type of oral or intravenous contrast medium is not further specified at all. When reading radiological literature on specific abdominal clinical problems, it is also evident that in the German-speaking world all types of hospitals and experts use different sequences and examination protocols for the same clinical problems. Overall, the inconsistency in radiology makes it difficult to use radiological examinations from different locations and institutions as comparable reference methods for major oncological or other medical studies.

The Abdominal and Gastrointestinal Imaging Study Group of the German X-Ray Association (Deutsche Röntgengesellschaft, DRG) pursues the goal of giving, based on evidence and in the near future, German radiology professionals the option of implementing the latest findings from radiology literature and using them for the purpose of improving and optimizing protocols. To create a type of "radiological guidelines" for imaging, it is first imperative to evaluate the current status in the German-speaking world. We therefore decided to address German radiologists, represented by the members of the Abdominal and Gastrointestinal Imaging Study Group as well as all members of the DRG as representatives of German radiology with an online survey on abdominal radiology to evaluate the current status of examination methods employed. The term "German-speaking world" was consciously chosen, since radiologists from Austria and Switzerland are also represented in the DRG and actively participated in the survey.

The results of the online survey and analyses with descriptive statistics are presented below following a brief introduction of the existing organ- and disease-focused guidelines and recommendations. For better readability and clarity, the decision was made to forgo using the standard division of the manuscript into a separate results and discussion section. Instead, these were critically discussed and evaluated according to bodily regions following an introduction of the results of the online survey.

This manuscript thus seeks to critically discus the reality of abdominal radiology in Germany, insofar as it is represented within the limits of an online survey, and contrast it with the ideal state or the state as recommended in current literature.

\section{Material and methods}

Following a preparatory discussion among the experts involved, an online survey was created using the SurveyMonkey $^{\odot}$ WebTool [16] (www.surveymonkey.com), the professional-level version of which was furnished by the German $\mathrm{X}$-ray Association. The survey consisted of 65 questions in total, 49 being multiple-choice question, 8 being worded as open-ended questions and another 8 being questions designed to elicit the proportional use of modalities through two numbers adding up to $100 \%$. The survey was published on the internet on July 17, 2014 and concluded on September 30, 2014, thus being available online for 76 days. The members of the Abdominal and Gastrointestinal Imaging Study group were invited to participate first through a personalized newsletter sent by the German X-ray Association. Two weeks later, all members of the German X-ray Association were invited and requested to participate in the survey through a DRG newsletter. The participants were advised that the survey would take approximately 15 minutes and was designed to evaluate the actual state of abdominal radiology in Germany. 
Statistical analysis was performed using the statistical tools implemented by SurveyMonkey ${ }^{\complement}$. Because the survey tool additionally allowed Excel and SPSS files to be generated, additional statistical evaluation was performed using Excel (Excel:mac 2011, Version 14.5.2, Microsoft, WA, USA). While in principle the survey was anonymous, the participants were asked to voluntarily indicate the name of their institution to avoid any repetitions.

\section{Results and discussion}

\section{Participants of the online survey}

Overall, the survey was started and partly completed by 103 participants. In the case of 13 participants, the survey was discontinued after just the first 4 questions, prompting us to exclude these participants with incomplete surveys from further analysis. This left a total of 90 participants who had completed at least $80 \%$ of the survey. Because none of the 65 questions posed were mandatory, individual questions could be skipped. Thus in the following evaluation, the absolute number of answering participants including the participants skipping the question is clearly stated at the outset.

Of the participants answering the survey, 27\% $(n=24)$ were private practice radiologists working at a medical office or a medical center. $4.5 \%$ were radiologists at a primary hospital, $24.7 \%(n=22)$ radiologists at a secondary hospital and $21.3 \%(n=19)$ at a tertiary hospital. $22.5 \%$ $(n=20)$ were radiologists at university hospitals (० Fig. 1). When the participants working in hospitals were asked about the number of beds at their facilities, the leading answer was more than 1.000 beds at $25.6 \%(n=21)$, followed by 401 to 600 beds at $15.8 \%(n=13)$. The survey was dominated by radiologists in managerial positions. Of the 90 participants answering the questions $15.5 \%(n=14)$ were owners or co-owners of the practice, while $11 \%(n=10)$ were employees of the practice. Of those working in hospi-

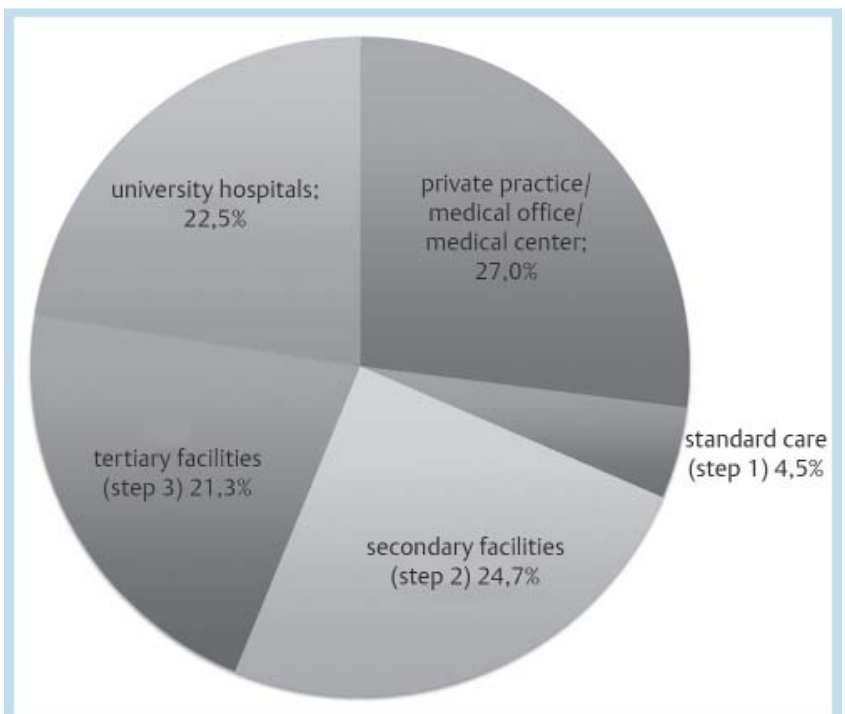

Fig. 1 Of the 90 survey participants, only $27 \%$ were employed in private practice (medical office/medical center). Of those working at hospitals, the participants were similarly distributed among university hospitals (22.5\%), tertiary $(21.3 \%)$ and secondary $(24.7 \%)$ facilities. tals, chiefs of radiology dominated at $28.9 \%(n=26)$, followed by senior physicians at $20 \%(n=18)$ and executive senior physicians at $12 \%(n=11)$. Residents likewise constituted $12.2 \%(n=11)$. While 55 of 90 participants indicated the name or location of the hospital or practice, 35 desired to remain anonymous.

\section{Esophagus and stomach}

Questions of the online survey pertaining to radiological esophageal and gastric imaging

Percentage of CT/MRI in staging esophageal/gastric cancer?
(total $100 \%$ )
Do you routinely administer antispasmodics for staging?
CT: Do you perform esophageal/gastric examinations with
distension of the esophagus/stomach?
CT: Positioning the patient?
CT: Which contrast medium phases are routine?
CT: What slice thickness reconstruction do you employ? $(\mathrm{mm})$
MRI: In which spatial planes do you examine?
MRI: Do you routinely use diffusion?
Does your RIS system have standardized text for generating
esophageal/gastric examination reports?

\section{Modalities, preparation and patient positioning}

The S3 guideline on esophageal (AWMF 2015 - register number 021 -0230L) and stomach cancer (AWMF 2011 register number 032 - 0090L) recommends for both entities a hydro-CT with spasmolysis as staging measure. MRI plays only a subordinate role for both entities [17].

Likewise according to the results of the survey, $89 \%$ of radiologists use computed tomography, while only $11 \%$ favor MRI ( $\bullet$ Table 1$)$.

The spasmolysis recommended in the guideline is performed by $65 \%$ of radiologists. The negative contrast (sometimes also referred to as "neutral contrast") with water for hollow organ distension is used by $62 \%$ ( $\bullet$ Table 2 ). A total of $22 \%$ use positive contrast medium, which can result in a worse presentation of the tumor-bearing wall portion as well as compromised evaluation of deep infiltration. In addition, a study by Ertuk et al. demonstrated significantly compromised evaluation of the gastrointestinal wall layers when a highly-positive oral contrast agent was employed [18]. Currently, $16 \%$ of radiologists do not perform distension. $93 \%$ of radiologists examine the patient in supine position, i.e. the prone posi-

Table 1 Preferred modality (CT, MRI) of the radiologist for the corresponding clinical problems and examination regions (results greater than $100 \%$ are due to double answers being possible in isolated cases).

\begin{tabular}{|c|c|c|}
\hline & $\mathbf{C T}$ & MRI \\
\hline esophageal/gastric carcinoma & $89 \%$ & $11 \%$ \\
\hline pancreatic carcinoma & $67 \%$ & $33 \%$ \\
\hline cystic pancreatic space occupations & $49 \%$ & $51 \%$ \\
\hline acute pancreatitis & $86 \%$ & $14 \%$ \\
\hline HCC, primary diagnosis & $49 \%$ & $51 \%$ \\
\hline CCC, primary diagnosis & $53 \%$ & $47 \%$ \\
\hline primary liver metastasis & $66 \%$ & $34 \%$ \\
\hline staging colon carcinoma & $83 \%$ & $24 \%$ \\
\hline staging rectal carcinoma & $31 \%$ & $75 \%$ \\
\hline
\end{tabular}


Table 2 Oral distension and contrasting for clinical problems in specific examination regions of the abdomen.

\begin{tabular}{llll} 
& no distension & $\begin{array}{l}\text { positive oral } \\
\text { contrast medium }\end{array}$ & $\begin{array}{l}\text { neutral oral } \\
\text { contrast medium }\end{array}$ \\
\hline $\begin{array}{l}\text { esophagus/ } \\
\text { stomach }\end{array}$ & $15.6 \%$ & $22.2 \%$ & $62.2 \%$ \\
\hline $\begin{array}{l}\text { pancreas } \\
\text { liver }\end{array}$ & $7.8 \%$ & $26.7 \%$ & $65.5 \%$ \\
\hline
\end{tabular}

tion recommended for better unfolding of the proximal portions of the stomach specifically for stomach cancer and gastric lymphoma $[19,20]$ is not widely accepted in clinical practice and is used by only $3 \%$ of radiologists.

\section{Technology}

The standard slice thickness is $3 \mathrm{~mm}$. Axial and coronal slicing is employed by nearly all institutes, while half the institutes additionally use sagittal reconstruction.

The contrast medium phases employed are listed in $\bullet$ Table 1. What stands out is that vascular arterial (=early arterial) phases are not implemented as well as the fact that most institutes forego non-contrast spiral. The biphasic protocol with parenchyma-arterial and portal vein phase is used by over $70 \%$ of radiologists.

\section{Reporting}

Only $20 \%$ of radiologists have access to reporting systems employing standardized text or similar solutions.

\section{Esophagus and stomach: discussion/summary}

Virtually all radiologists currently perform computed tomography for staging esophageal and stomach cancer in full conformity with the guideline. A majority of over $60 \%$ also uses negative contrast (neutral contrast) for wall distension while simultaneously employing spasmolysis.

\section{Pancreas}

Questions of the online survey pertaining to radiological imaging for the pancreas and biliary ducts

Primary pancreatic cancer staging. What is your ratio of CT to MRI
(total $100 \%$ )?
Primary imaging of "cystic tumors of the pancreas": What is your
ratio of CT to MRI (total $100 \%$ )?
Primary examination of pancreatitis: What is your ratio of CT to MRI
(total $100 \%$ )?
CT: Do you perform oral contrasting/ distension of the stomach?
CT: Which phases do you employ in cases of pancreatic cancer?
CT: Which phases do you employ for pancreatitis?
CT of the pancreas: Positioning the patient?
CT of the pancreas: What slice thickness (mm) do you use for
reconstruction?
MRI of the pancreas: In which spatial planes do you perform
examination?
MRI: Do you routinely use diffusion in the pancreas protocol?
MRI: Do you routinely use MRCP sequences in the pancreas
protocol?
MRI/MRCP: Do you administer oral contrast medium when
performing MRCP?
MRI/MRCP: Do you perform secretin-enhanced MRIs?
Does your RIS system have standardized text for generating
pancreas examination reports?

Modalities, preparation and patient positioning

The recommendations of the S3 guideline for exocrine pancreatic cancer (AWMF 2013 - register number 032-0100L) for clarifying a suspected tumor as well as for evaluating the spread of pancreatic cancer originate from 2006. When the recommendations were updated in 2013, the imaging were not revised but rather remained the same as in the 2006 version [13]. In the survey, this heterogeneity was reflected in CT and MRI being used for staging in $67 \%$ and $33 \%$ of cases, respectively ( $\bullet$ Table 1 ). For diagnosing the spread of cancer, the guideline recommends MDCT (multi-detector CT) and endosonography, designating MRI as optional and allowing other methods to be bypassed when CT is available.

In the case of cystic tumors of the pancreas, upper abdominal sonography, MDCT, MRI with MRCP, endosonography and ERCP can equally be used for differential diagnosis, even if a definitive diagnosis cannot be issued according to the guidelines. A sound statement can be made only in connection with clinical findings or if methods are combined with one another. This heterogeneity is likewise reflected in the survey with CT and MRI being used in $49 \%$ and $51 \%$ of cases, respectively, for clarifying cystic tumors of the pancreas.

Acute pancreatitis is clarified by $86 \%$ of radiologists using CT and only in $14 \%$ cases through MRI ( $\bullet$ Table 1 ). While there is no AWMF guideline for acute pancreatitis, a publication appeared in 2007 in Internisten und Deutschen Ärzteblatt on evidence-based imaging and therapy by Huber et al., who favored CT in a 7 to 10-day interval, recommending it with the word "should" [21]. CT is recommended in the early phase only when therapeutic consequences are expected (puncture, drainage). MRI is also presented as an alternative with low side effects.

Distension of the stomach for improved imaging of the pancreas is performed by $66 \%$ of all radiologists and is thus employed at a slightly higher rate than when diagnosing the esophagus and stomach ( 0 Table 2 ). When imaging the pancreas, $27 \%$ use positive, oral contrast media, while $8 \%$ use no oral contrast media whatsoever.

Examining the patient in supine position is preferred by $82 \%$, while $14 \%$ prefer side/oblique positioning.

\section{Technology}

For imaging the pancreas, standard thickness for axial reconstruction is $3 \mathrm{~mm}$. While axial and coronal slicing is performed at nearly all facilities, approximately $60 \%$ of the survey participants additionally use sagittal reconstruction. The standard contrast medium phases differ only slightly with regard to pancreatic cancer and pancreatitis ( $\bullet$ Table 3 ). Because, according to the $7^{\text {th }}$ edition of the TNM Classification, the key distinguishing feature between the T3 and T4 stage is the infiltration into the arterial vascular path, an additional arterial phase, which is also performed by $77 \%$ of those surveyed, is also accorded central importance [22]. Although the non-contrast phase can provide additional information in cases of pancreatitis, it is employed by $34 \%$ of radiologists just as frequently as when diagnosing cancer. When MRI is used as a modality, already two thirds of all radiologists now routinely perform diffusion sequences, which suggests the potential of functional imaging, even if it is not yet currently standard repertoire and different approaches for pancreas diffusion are employed [23]. 


\begin{tabular}{|c|c|c|c|c|c|c|}
\hline & $\begin{array}{l}\text { non-contrast } \\
\%\end{array}$ & $\begin{array}{l}\text { early arterial } \\
\%\end{array}$ & $\begin{array}{l}\text { arterial } \\
\%\end{array}$ & $\begin{array}{l}\text { portal venous } \\
\%\end{array}$ & $\begin{array}{l}\text { late } \\
\%\end{array}$ & $\begin{array}{l}\text { DWI } \\
\%\end{array}$ \\
\hline esophageal/gastric carcinoma & 14 & 6 & 71 & 97 & 7 & 70 \\
\hline pancreatic carcinoma & 33 & 31 & 77 & 100 & 8 & 67 \\
\hline acute pancreatitis & 34 & 9 & 60 & 97 & 7 & 67 \\
\hline hepatocellular carcinoma (HCC) & 38 & 39 & 68 & 98 & 10 & 77 \\
\hline cholangiocellular cancer (CCC) & 29 & 22 & 76 & 100 & 34 & 77 \\
\hline liver metastases & 19 & 13 & 74 & 100 & 8 & 77 \\
\hline colorectal carcinoma & 14 & 7 & 51 & 98 & 6 & 49 \\
\hline
\end{tabular}

Table 3 Routinely acquired contrast phases for specific clinical problems and tumors.

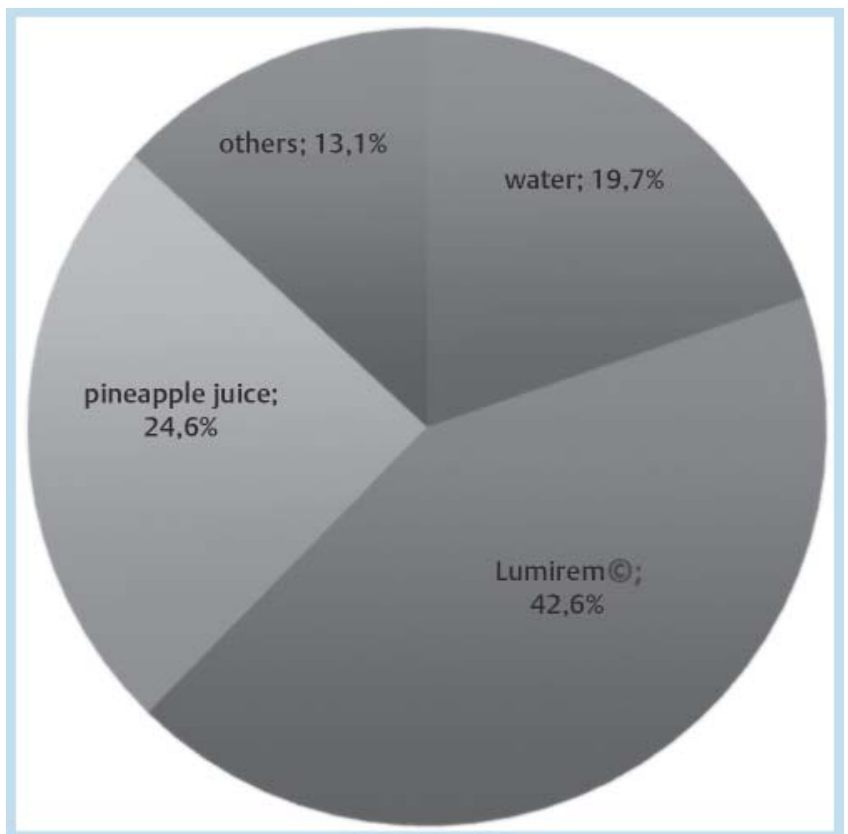

Fig. 2 Answers to the question, "Do you administer oral contrast for MRCP?" The question was answered by 61 of 90 survey participants. The largest group of radiologists (42.6\%) answered that they administer oral contrast medium in the form of Lumirem ${ }^{\circledR}$, an paramagnetic substance containing iron oxide for reducing T2 signal, which is currently no longer available on the market.

For $84 \%$ of radiologists, MRCP is part of the routine examination protocol for imaging the pancreas and in many cases is performed with additional oral contrast media ( $\bullet$ Fig. 2 ). MRCP is thus not used by $16 \%$ of radiologists. One third of the survey participants additionally offer secretin-enhanced MRI.

\section{Reporting}

The trend toward standardized reporting has not yet made its way into the imaging of the pancreas or the upper GI tract in general, and only $18 \%$ of radiologists have standardized text for pancreatic imaging in their RIS.

\section{Pancreas: Discussion/summary}

Despite a lack of updated recommendations for pancreatic cancer imaging, there are clear standards for imaging the pancreas, which are followed by the majority:

CT examination with distension of the stomach using negative contrast medium with the patient positioned on his or her back or obliquely and the use of a triphasic protocol with non-contrast, arterial and portal venous phase. MRI is used primarily with MRCP and oral administration of contrast medium. Secretin-enhanced MRI is no longer offered uniformly or universally. In the meantime, diffusion sequences have become part of routine protocol for MRI. Standardized text for generating pancreatic imaging reports has currently not yet become an established standard.

\section{Liver}

Questions of the online survey pertaining to radiological imaging of the liver

CT: Do you administer oral contrast medium when performing CT
examinations for liver space occupations (abdominal CT staging)?
CT: At what slice thickness do you reconstruct? (mm)
CT: Which routine reconstructions do you save in PACS?
Primary HCC staging. What is your ratio of CT to MRI (total 100\%)?
CT: What phases do you run when performing HCC?
MRI: Do you routinely administer liver-specific contrast media
when performing HCC?
Primary CCC-staging. What is your ratio of CT to MRI examinations
(total $100 \%$, please)?
CT: Which phases do you run when performing CCC?
MRI: Do you routinely administer liver-specific contrast media
when performing CCC?
Primary liver metastases staging: What is your ratio of CT to MRI
(total $100 \%$ ?
CT: Which phases do you run for liver metastases?
MRI: Do you routinely administer liver-specific contrast media in
Cases of liver metastases?
MRI, general liver: On which spatial planes do you examine the liver?
MRI, general liver: Do you routinely use diffusion sequences when
imaging the liver?
Does your RIS system have standardized text for generating liver
tumor reports?
As a radiologist, do you perform locally ablative measures for liver
tumors?
Is TACE performed at your facility?
Does your RIS system have standardized text for reporting on space
occupations of the liver?
How is PTCD performed at your facility?
Who performs CEUS (contrast-enhanced ultrasound) for space
occupations of the liver?

\section{Modalities, preparation and patient positioning}

The latest version of the S3 guideline for the diagnosing and treatment of hepatocellular cancer (HCC) was published in 2013 (AWMF 2013 - register number 032 -0530L) [4, 14, $24,25]$. With regard to imaging patients at high risk, the guideline mentions arterial hypervascularization with rapid washout of the contrast agent and relative contrast inversion with the surrounding liver parenchyma. In the same section it is recommended that this contrast pattern be recorded with a triphasic contrast-enhanced sectional image 
methods without addressing the value of CT, MRI or contrast-enhanced ultrasound (CEUS). Contrast-enhanced MRI (evidence level Ia) is recommended for diagnosing the spread in the sense of intrahepatic metastasis, while a thoracic CT (Good Clinical Practice, GCP) is advised for diagnosing extrahepatic spread. For follow-up care, multi-phase sectional imaging, preferably MRI (GCP), is recommended. When evaluating response to local chemotherapy (TACE), a contrast-enhanced CT or MRI is recommended no earlier than 4 weeks and no later than 3 months following TACE. The guideline does not address the value of liver-specific contrast medium. Additionally, there is no further mention or discussion in the guideline regarding the performance of diffusion-weighted sequences. There are no recommendations for performing examinations using CT or MRI. The performance of a late-arterial phase as required in LI-RADS, for example, is given no further consideration in the German guideline [26, 27].

Because there are no comprehensive current German guidelines for evaluating cholangiocellular cancer (CCC) [28, 29], it is not possible to adequately contrast actual practice with official recommendations.

The queried spread of locally ablative and chemotherapy methods (radio frequency ablation (RFA) and transarterial chemoembolization) for HCC are also recommended in the guidelines as bridging therapy for downstaging. Regarding TACE, the guideline states that it should be used for patients for whom no curative method is available and who have stage CHILD-Pugh A or B solitary or multifocal HCC without extrahepatic metastasis. In this respect, it is noted that an indication for TACE must be issued by an interdisciplinary tumor board.

With regard to the modalities for primary HCC staging, CT and MRI are used nearly equally according to the $87 \mathrm{com}-$ pleted answers, with 49 for CT and 51 for MRI ( 0 Table 1 ). For contrast medium phases, a non-contrast examination still performed anyway in nearly $38 \%$ of cases and an early arterial phase in likewise $39 \%$ of cases. The late arterial phase, which is required according to the LI-RADS recommendations is performed by a majority of $68 \%$. Nearly all participants examine the portal vein phase, while $40 \%$ of radiologist perform a late or parenchymal phase (0 Table 3 ). When examining space occupations of the liver, $28 \%$ routinely provide no oral contrast medium whatsoever, while $54 \%$ administer a positive contrast medium and $18 \%$ a negative contrast medium ( $\bullet$ Table 2 ).

Coronal and axial slices are taken nearly $100 \%$ of the time and saved in the PACS, while sagittal reconstructions are documented in only $65 \%$ of cases. The use of liver-specific contrast medium when examining HCC is interesting, with $25 \%$ of those surveyed (88 answers) routinely using no liver-specific contrast medium, while over $70 \%$ administer Gd-EOB-DTPA (Primovist, Bayer Healthcare). Just under 5\% surveyed routinely use Gd-BOPTA (Multihance, Bracco) when initially assessing HCC.

When it comes to primary evaluation and primary staging of CCC, a situation similar to that of HCC appears in the distribution of modalities, with $53 \%$ of those surveyed using $\mathrm{CT}$ and $47 \%$ using MRI in the first instance. A similar distribution appears with respect to the contrast medium phases for evaluating CCC, with $29 \%$ routinely using non-contrast, $22 \%$ early arterial and $76 \%$ a late arterial phase. All survey participants perform a portal venous phase. However, the late phase, which the literature actually recommends for characterizing and detecting CCC, is only used routinely by $34 \%$ [ $30-32$ ]. A total of $39 \%$ primarily do not use liver-specific contrast medium when assessing CCC. Gd-EOB-DTPA is routinely used by $56 \%$ and Gd-BOPTA by $5 \%$.

The survey question concerning the choice of modality for performing primary staging of liver metastases yielded another picture. In this case, $66 \%$ routinely use CT as standard method, while only $34 \%$ routinely use MRI as primary method ( $\odot$ Table 1 ). With regard to choice of contrast medium phase, all participants perform a portal venous phase, with $74 \%$ performing a late arterial phase in any case. Noncontrast examinations and early arterial phases are the exception if anything, being used by $74 \%$ and $19 \%$ of surveyed, respectively ( $\bullet$ Table 3 ). When asked about the evaluation of liver metastases during initial imaging, $48 \%$ primarily use no liver-specific contrast medium, but rather $48 \%$ use GdEOB-DTPA when performing an initial MRI and $5 \%$ Gd-BOP-

TA when rescanning

The liver is examined principally on the axial and coronal planes, with only $23 \%$ additionally performing sagittal MRI sequences.

$77 \%$ of surveyed indicate routinely using diffusion-weighted sequences for evaluating space occupations of the liver. As in the case of pancreatic imaging, this represents a positive and innovative application of this new technology that already appears to be routine in German-speaking countries even before being adopted into a guideline.

Regarding the question of structured reporting and the existence of standardized text for generating liver tumor reports, only $17 \%$ answered that they had access to pre-authored standardized text for generating reports.

\section{Therapeutic liver interventions}

$90 \%$ of surveyed perform CT-guided local ablative measures. With multiple answers being permitted, $22 \%$ indicated also using ultrasound for planning therapy. Radiofrequency ablation (RFA) is most common at $91 \%$, followed by microwaves at $38 \%$. Only $13 \%$ of those surveyed routinely perform IRE (irreversible electroporation), followed by $7 \%$ offering brachytherapy of the liver. Regarding local chemoembolization, $57 \%$ report offering TACE.

When asked who at their respective hospital performs percutaneous transhepatic cholangiodrainage (PTCD), only $54 \%$ of those surveyed indicated that this intervention is performed by the radiology department. Of the 71 total answers, with multiple answers being permitted, $62 \%$ indicated that this examination/intervention is performed by their hospital's gastroenterology department, with only $4 \%$ reporting that it is performed their colleagues in surgery.

\section{Ultrasound examination of the liver}

There is likewise an interesting distribution pattern when it comes to the performance of contrast-enhanced ultrasound (CEUS) ( $\bullet$ Fig. 3 ). With multiple answers being permitted and a total of 74 answers being provided, CEUS is performed in $94.6 \%$ of cases $(n=70)$ by the gastroenterology department. Only $24.3 \%(n=18)$ of the surveyed radiologists answered that CEUS is also performed in the radiology department. Only $7 \%$ perform CEUS in the 


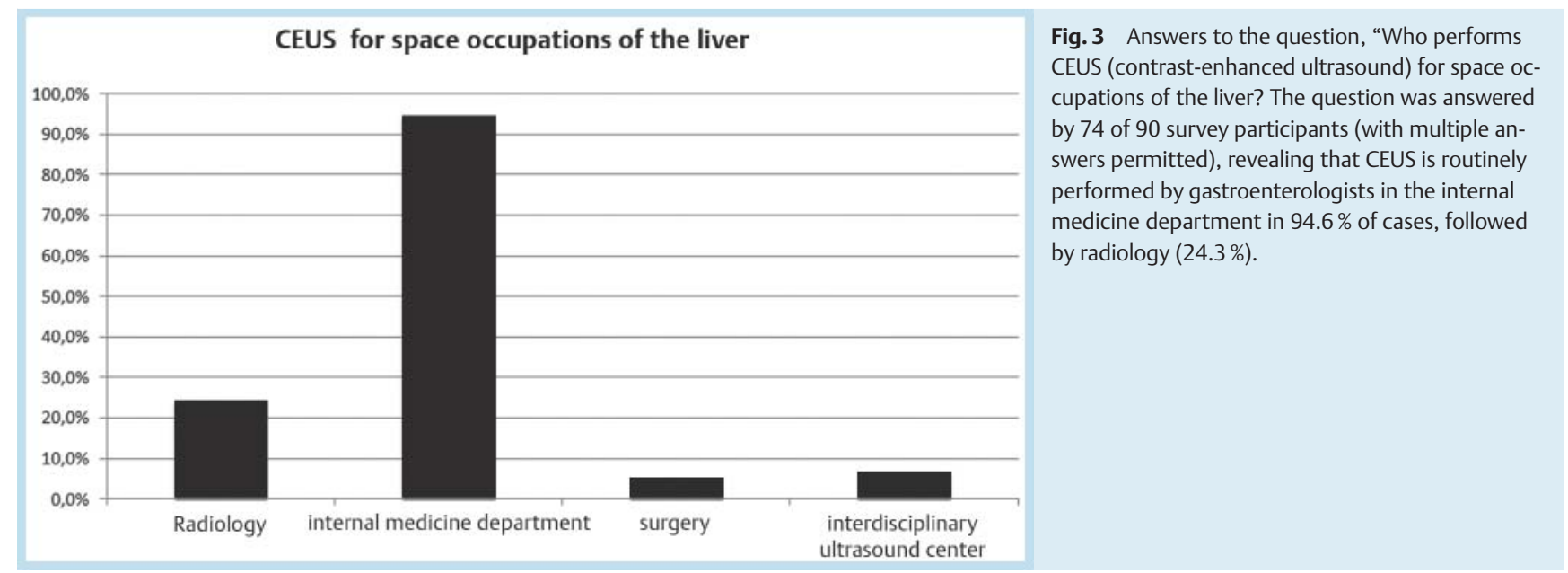

interdisciplinary ultrasound centers with radiologists being involved and $5 \%$ in the surgery department.

\section{Discussion: Liver}

The German S3 guideline for diagnosing and treating HCC is relatively vague when it comes to performing examination and provides no recommendations whatsoever on how to perform CEUS examinations as well as CT and MRI. As such, it does not clearly define arterial phase, which, in the LI-RADS recommendations is unambiguously late arterial phase. The guidelines do not further address the administration of liver-specific contrast media or the performance of diffusion-weighted sequences for diagnosing space occupations of the liver. Thus the diagnostic value of early arterial phase for CCC indicated by $1 / 5$ of those surveyed and the early arterial phase for HCC indicated by $1 / 3$ is highly controversial. According to evidence, the performance of non-contrast phases would also appear to be questionably useful. It is also astounding that of the surveyed, only $40 \%$ perform late phase for HCC and $34 \%$ for CCC, which is frequently of critical diagnostic importance for the respective tumors $[4,32,33]$. It is surprising that over $75 \%$ of the radiologists surveyed routinely administer liver-specific contrast media for evaluating space occupations of the liver, even though the guidelines provide no evidence in this regard. In principle, however, it can be assumed, based on current literature, that the use of liverspecific contrast medium as well as diffusion-weighted sequences will certainly be increasingly important for future guidelines. It must be viewed critically that for new and developing methods such as LI-RAD, the image signals from liver lesions with regard to liver-specific contrast medium and diffusion signal are presently given subordinate priority (minor criterion for LI-RADS) [26, 27].

The answers provided by the surveyed radiologists in German-speaking countries concerning the imaging of the liver are characterized by a relative inhomogeneity. It is precisely in this highly sensitive area, which is not discussed in further detail in the guidelines and in which only generally triphasic sectional imaging methods are recommended, that radiologists urgently need to make changes based on evidence and provide more specific recommendations for imaging.

\section{Small intestines}

Questions of the online survey pertaining to radiological imaging of the small intestines with regard to chronic inflammatory intestinal diseases

Which small intestinal examinations do you routinely perform?
Do you perform conventional enteroclysis, and if so, how many
per year?
How do you distend the small intestines in CT or MRI (enteroclysis
or enterography)? What is your routine procedure?
Do you perform additional rectal administration of contrast medi-
um for CED patients when performing CT/MRI?
MRT: Do you perform diffusion-weighted imaging for CED?
Who performs intestinal ultrasound at your facility?

\section{Modalities}

For examining the small intestines, the S3-guideline "Diagnosing and treating Morbus Crohn" (AWMF 2014 - register number 021 -004) last revised in 2014 above all plays a decisive role in indicating imaging $[34,35]$. The guideline dictates that patients exhibiting clinical signs of chronic inflammatory intestinal disease first undergo high-resolution transabdominal sonography and ileocolonoscopy with serial biopsy. Critical, however, is the point for the initial imaging of the small intestines in which performing an MRI of the small intestines (enterography or enteroclysis) is recommended. For the further course of the disease, sonography is recommended as primary method for evaluation of activity. If visibility is compromised, however, the revised version of the guideline recommends an endoscopy or MRI depending on the primary localization. As in the previous version of the guideline, a conventionally performed enteroclysis under fluoroscopy is no longer recommended or no longer mentioned as primary modality. To limit radiation exposure, the updated version of the S3 guideline if anything even downgrades the use of CT for evaluating the small intestines. The current version goes so far as recommending high-resolution sonography or MRI with only low evidence level (evidence level I) for diagnosing extramural complications, such as fistulas and abscesses, reserving, in accordance with the new guideline, computed tomography only for emergencies or when 
other diagnostic methods prove ineffective to limit radiation exposure.

With regard to the question concerning which examinations of the small intestines are routinely performed, 88 of 90 questionnaires were fully completed. With multiple answers being permitted, $95.5 \%$ indicated that they examine the small intestines with MRI, followed by $34 \%$ reporting that they use CT. Conventional enteroclysis is performed by $20 \%$ of the surveyed. It can thus be concluded that MRI of the small intestines is offered in nearly all cases, while CT of the small intestines and conventional small intestinal examination are regularly offered and performed in the program. The open-ended question as to how many conventional enterograms per year were still performed was answered by 34 participants with a value between 0 and 50 per year. Thus, an average of 8.5 examinations are performed per year, with the median being 4 . Of the 4 answering institutions performing more than 25 conventional small intestinal examinations annually, two facilities were a medical center (25 and 50 examinations per year), while the other two were each a Swiss and German university hospital performing 50 and 30 examinations per year, respectively.

The guideline takes no position whatsoever regarding the issue of correct distension during a CT or MRI small intestinal examination Both methods (enterography and enteroclysis) are merely mentioned.

The question, "How do you distend the small intestines in CT or MRI (enteroclysis or enterography)?" was intended to evaluate which portion enterography and enteroclysis constitute among the sectional imaging methods. In 86 of the 90 questions answered (with multiple answers being permitted), the use of oral contrast medium for imaging the small intestines (enterography) dominated at $86 \%$ $(n=74)$ followed by enteroclysis via a nasojejunal probe at $22 \%(n=19)$. Of the 90 study participants, 87 answered the question concerning additional rectal administration of contrast medium in patients with chronic inflammatory intestinal diseases when performing CT or MRI of the small intestines, with $57 \%$ reporting that they do not additionally administer contrast medium rectally on a routine basis (multiple answers were not permitted). The question concerning routine use of diffusion-weighted imaging in the case of chronically inflammatory intestinal diseases was answered by 86 of the 90 participants, with $45 \%$ reporting that they routinely perform a DWI sequence.

The current guideline explicitly mentions high-resolution intestinal ultrasound as a primary method to be used during initial diagnosis, but also during the course of the disease as well as for evaluating activity. From a radiological point of view, it is also clearly important for reasons of professional policy who routinely performs this high-resolution intestinal examination.

This question was answered in 78 of the 90 questionnaires, revealing that high-resolution intestinal ultrasound is performed in the majority of cases by gastroenterologists/internists $(76 \%(n=59))$. In $13 \%$ of cases, high-resolution intestinal ultrasound is performed at an interdisciplinary ultrasound center, and in $11 \%$ of cases it was offered by the radiology department.

\section{Discussion: Small intestines}

In the survey, the majority of radiologists reflected procedure that is primarily in conformity with guidelines, with over $95 \%$ indicating MRI of the small intestines as intestinal examination. Of the $34 \%$ who perform intestinal CT, in the majority of cases this was their second answer, which merely showed that they also offer this modality. However, based on the most current guideline from 2014, radiologists need to proceed more cautiously with regard to limiting radiation exposure among the frequently very young patients with chronic inflammatory intestinal diseases.

The distribution of answers regarding the type of distension, i.e., enteroclysis or enterography looks interesting, which in recent years have probably led to a strong change in favor of exclusively oral administration of contrast medium (enterography) at $86 \%$. The fact that $55 \%$ answered affirmatively to the routine use of diffusion-weighted imaging in MRI for chronic inflammatory intestinal diseases must be viewed very positively. While this method is clearly still new and has not yet been decisively evaluated in the literature, the progressive use of this promising functional MR method for evaluating inflammatory changes without the use of intravenous contrast medium is seen in the German-speaking countries.

\section{Colon and rectum}

Questions of the online survey pertaining radiological examination of the colon and rectum

Do you regularly perform virtual colonographies?
If yes, how many virtual colonographies do you perform annually?
Which special methods do you employ when performing virtual
colonography?
How do you normally stage colorectal cancer?
How do you normally stage (CT/MRI) rectal cancer locally?
(total $100 \%$ )?
Do you normally perform diffusion-weighted imaging in cases of
rectal cancer?
In your routine reports for rectal cancer, do you normally mention
the distance from the mesorectal fascia?
CT: Which contrast medium phases do you use when performing
CT in cases of colorectal cancer?
Do you routinely perform conventional contrast-enhanced
(fluoroscopy) examinations of the colon?

\section{Early detection of colorectal cancer/incomplete colonoscopy}

The AWMF S3 guideline for colorectal cancer [36] last revised in August 2014 once again emphasizes the importance of conventional colonoscopy as the method with the highest sensitivity and specificity when it comes to early detection and preventative care (level of evidence 3b, degree of recommendation $\mathrm{B}$ ). In contrast, this guideline states that neither CT colonography nor MR colonography should be used for intestinal cancer preventative care/early detection in the asymptomatic population. However, the guideline also asserts that a CT or MR colonography should be performed in cases of incomplete colonoscopy (e.g., adhesions) or if the patient still wishes to have a complete assessment of the colon (level of evidence $3 \mathrm{~b}$, degree of recommendation B, strong consensus). On the other hand, there is no longer any mention of the double-contrast ex- 
amination. Endoscopically impassible stenosis constitutes a special situation. In the event of an incomplete colonoscopy due to a stenotic tumor, the guideline additionally recommends a CT or MR colonography prior to surgery (degree of recommendation $B$, level of evidence 4). Because the value of preoperative colon contrast uptake is reportedly of limited value and, in the case of stenosis, is associated with the risk of inducing ileus, it is not recommended in the guideline.

Of the 90 professionals surveyed, 88 answered the question regarding whether they regularly perform virtual colonography, with $48.9 \%$ answering in the affirmative and $47.7 \%$ answering in the negative. MR colonography is performed by only $3.4 \%$ of those surveyed. No exact information was provided as to the frequency of virtual colonography (examinations per month). The comparably low use of MR colonography reflects the clearly lower body of data in a scientific sense as well.

The recommendations regarding indications, technical performance and evaluation of virtual colonography are based on the consensus statements of the ESGAR (European Society Gastrointestinal and Abdominal Radiology) in the most recent version dated 2013 [37] as well as a consensus paper between the ESGAR and the ESGE (European Society of Gastrointestinal Endoscopy) as appearing in 2014 [38]. Use of "3 D technologies" is accordingly mandatory when evaluating virtual colonography. However, it is at the user's discretion as to which of the commercially available technologies (so-called fly-through, colonic dissection, filet view, panoramic view etc.) are to be used.

Of the 90 participants, 53 ( $\bullet$ Fig. 4 ) provided answers regarding the specific technologies of virtual colonography. Consistent with the consensus statements, $3 \mathrm{D}$ technologies for evaluation are widely distributed. $92.5 \%$ of the surveyed use in particular so-called "fly-through" technologies analogously to conventional intestinal endoscopy. So-called "colon dissection", i.e. the evaluation of virtually dissected intestinal halves, is preferred by $47.2 \%$. CAD programs (computer-assisted diagnosis) may be used according to the specifications of the ESGAR as so-called "second-reader", yet as such do not replace the primary image report issued by the radiologist. CAD is regularly used by $26.4 \%$ of the surveyed.

The issue of "conventional contrast-enhanced examinations of the colon" was addressed by 86 of the 90 professionals surveyed, with $47.7 \%$ answering that they do not perform conventional contrast-enhanced examinations of the colon. When contrast-enhanced examinations of the intestines are performed, $40 \%$ are mono-contrast examinations, while a smaller portion of $19.8 \%$ are double-contrast examinations. The high percentage of mono-contrast examinations may reflect the overwhelming use of contrast-enhanced examinations of the colon as a process for excluding anastomosis insufficiencies, stenosis, etc. to answer questions that tend to be more surgical in nature.

\section{Preoperative local findings and diagnosing the spread of colorectal cancer}

With regard to preoperative staging for diagnosing the spread of cancer, the S3 guideline recommends a multiline abdominal CT in addition to primary abdominal sonography when suspicious findings are present, the liver has compromised visibility on ultrasound, or liver metastasis is suspected based on clinical findings. It is also established that multi-line CT is best suited particularly for evaluating the resectability of liver metastases (locating the metastases in relation to the hepatic veins, the hilar structures as well as the vena cava). On the other hand, the extent of the liver metastasis is best assessed by MRI.

For local staging of colorectal cancer, the S3 guideline recommends multi-line CT for differentiating between tumors with and without infiltration of the wall. It concedes, however, the limited success in identifying nodal status.

The question regarding diagnosing the spread of and locally staging colorectal cancer was answered by 87 of the 90 participants, showing a clear preference for CT (83\%) over MRI (24\%). This response underscores the value of CT as the method of choice in the preoperative staging of colorectal cancer.

The S3 guideline does not provide specific information on slice thickness, oral/rectal preparation or even the i.v. administration of contrast medium/contrast phases.

According to the survey, $59.8 \%$ of participants administer contrast medium rectally, with $26.4 \%$ additionally giving i.v. Buscopan. While the overwhelming majority of those surveyed (98\%) acquire image data in the portal venous phase, $51 \%$ gather an additional data set in the arterial phase ( Table 1 ). In addition, $14 \%$ prefer prior non-contrast imaging, and $6 \%$ acquire late phases.

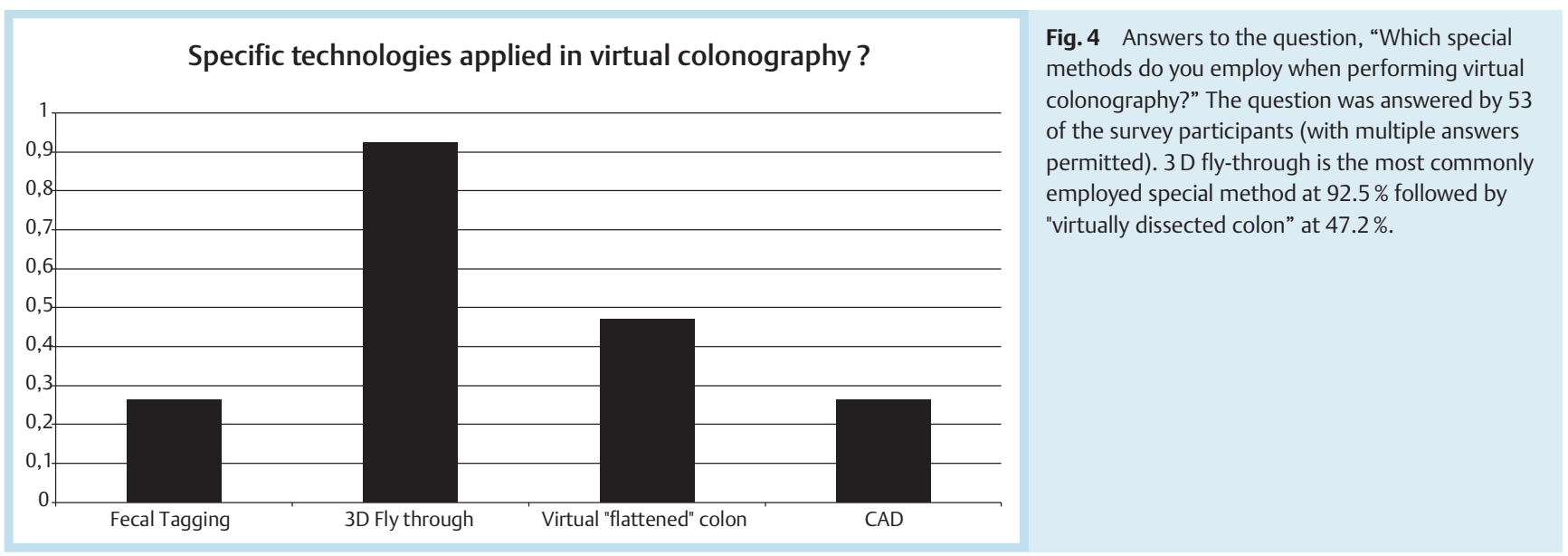


Preoperative local findings for rectal cancer

According to the guideline, MRI should preferably be performed for the local staging of rectal cancer, and endosonography in the case of presumed $\mathrm{T} 1$ cancer (degree of recommendation $B$, level of evidence $2 \mathrm{~b}$ ). In this context, $\mathrm{CT}$ is viewed more as an alternative method of limited accuracy in evaluating tumors in the bottom third versus the upper two thirds and unsuited for evaluating T1 tumors (level of evidence $2 \mathrm{~b}$ ).

The survey question regarding local staging of rectal cancer yields an opposite picture than that of the staging of the other colorectal cancers. Only a $31 \%$ minority of the 83 total answering participants "stages" rectal cancer with CT, while $75 \%$ are consistent with the guideline and thus prefer MRI.

According to the S3 guideline, MRI currently provides the greatest sensitivity and specificity for presenting the mesorectal fascia and the relative position of the tumor. Spiral CT constitutes a possible alternative, while endosonography does not show the fascia. On the other hand, the guideline does not address dedicated techniques, in particular multiparametric MRI.

According the survey, 58\% use "diffusion imaging" for local staging of rectal cancer. The distance of the tumor from the mesorectal fascia is indicated by $89.5 \%$ when performing initial MRI. This portion is much lower at $27.9 \%$ when CT is performed for staging rectal cancer. $8.1 \%$ do not indicate distance of the tumor from the mesorectal fascia whatsoever regardless of imaging technique.

\section{Discussion: colon and rectum}

While the general field of radiology frequently views virtual colonography as an "exotic" method employed only by select centers, the survey shows that this method is used regularly and according to indication by half of the participants surveyed. Fortunately, there is also broad and consistent use in this area of the evaluation techniques recommended by the professional associations. As is the case with many conventional hollow organ examinations, a trend from double toward mono-contrast is also occurring with contrastenhanced examinations of the colon. The nevertheless relatively high portion of double-contrast examinations (approximately $20 \%$ ) may come as somewhat of a surprise. It would appear that colleagues with years of experience in double-contrast examinations continue to employ this method. The guidelines and consensus statements issued by the professional associations clearly rank virtual colonography as the alternative method of choice when conventional colonoscopy is incomplete or infeasible, also recommending that it be used as primary method insofar as it is indicated. To a broad extent, the local staging and diagnosing of the spread of colorectal cancer are overwhelmingly performed through $\mathrm{CT}$ in conformity with the guidelines. However, there is heterogeneity when it comes to acquisition of contrast medium phases. Further standardization is needed in this respect, particularly also for limiting radiation exposure. For rectal carcinoma, MRI is preferred, likewise in conformity with guidelines. However, the guideline does not exclude the use of CT as an alternative. The distance from mesorectal fascia is not regularly described when CT is performed (approximately 30\% compared to $90 \%$ for MRI). Additional measures for structured reporting of rectal carcinoma are needed in this regard.

\section{Discussion and summary}

\section{$\nabla$}

The online survey conducted by the Abdominal and Gastrointestinal Imaging Study Group impressively shows that the apparent gap between guideline-compliant imaging and the actual practice in the field of abdominal radiology in German-speaking countries is actually smaller than believed.

Even if a somewhat restrained approach is taken in interpreting the results given the limited number of participants, the overwhelming majority of participating radiologist follow the guidelines in most cases when it comes to the entire gastrointestinal tract (esophagus, stomach, small intestines, colon and rectum) including the pancreas. The caveat would certainly be that because of the recentness of the pancreatic cancer guideline, the "imaging" have not been revised and have thus remained the same since 2006. The vast body of new publications makes a new edition an urgent goal. On the other hand, the evaluation of the implementation of the liver S3 guideline for hepatocellular cancer would appear to be problematic. This German S3 guideline remains vague on performing examinations, and there are additionally no recommendations regarding the exact performance of CEUS examinations or CT and MRI examinations. A radiological revision as well as updated information regarding the diagnosis and interventional treatment of liver cancer would be in order here.

With regard to imaging the small intestines, most radiologist follow the guidelines and thus use MRI. A noteworthy development is the fact that oral administration of contrast medium (MR-enterography) is currently the most commonly performed method at $86 \%$. It would also be worth mentioning that high-resolution intestinal ultrasound, which the guidelines specify as primary imaging tool, is entrenched more in gastroenterology than in radiology, being performed within the former field $76 \%$ versus $11 \%$ of the time for the latter.

Regarding the colon, it is surprising that nearly half of the radiologists participating in the survey regularly perform CT-based virtual colonography. When staging colon cancer, most radiologists follow the guideline, with CT examinations dominating. However, there is considerable divergence regarding the various contrast phases. Here as well, action is necessary for achieving uniformity in the contrast protocols and contrast phases.

In terms of methodology, our evaluation must be viewed critically given the fact that 90 valid completed online questionnaires certainly cannot constitute a true representation of all German radiologists. However, this number of completed forms alone must be viewed as a positive, given the relatively detailed nature of the questionnaire, which was completed on an absolutely voluntary basis, requiring on average over 15 minutes. It is also worth noting the dominating portion of radiologists in managerial positions in medical offices and hospitals, who were motivated alone by the questions being posed and who valued the survey more as a result of the answers providing an overview of their institute or medical office. In addition, it must be assumed that these questionnaires were completed by radiologists focusing particularly on the abdomen and thus do not represent the broad treatment reality, but rather reflect interested and motivated radiologists. 
Finally, it is necessary to critical address the creation and maintenance of guidelines from the viewpoint of radiologists in Germany. On a positive note, it must be mentioned that adequate interdisciplinary participation of every important medical specialization can be assumed for the S2 and S3 guidelines in Germany. However, the technical performance of radiological examinations is not the focus of these guidelines, which are read and implemented primarily by the general surgical and non-surgical specializations treating patients directly. Nevertheless, there has been a shift in the significance of applying the guidelines in radiology as well as a result of a vast number of publications concerning guideline implementation addressed to radiologists $[9,34,39-42]$.

In summary, it can be established that particularly with regard to radiological examinations of vague or outdated guidelines such as those for exocrine pancreatic carcinoma, for example, but also in the case of hepatocellular carcinoma, sectional imaging in the German-speaking countries is performed relatively non-uniformly and, in terms of contrast phase, frequently not in conformity with current radiological literature. Contrast phases that are sometimes unnecessary and supported by barely any current literature are performed, which clearly allows further room for improvement in terms of radiation safety. At this point, it is once again critical to reassert the radiologist's duty to, when faced with methods of equal value, favor of the method subjecting the patient to no or less radiation exposure. In several guidelines, CT or MRI, for example, are mentioned and offered as being parallel to one another. However, MRI should be used here when possible, given its superior sensitivity and absence of radiation. This applies especially for clarifying the small intestines, but also for staging rectal carcinoma or HCC. Overall, however, the examination behavior of most study participants when it comes to new radiological technologies such as diffusion-weighted imaging or liver-specific contrast media can be characterized as being very progressive and open to new technologies.

The results of the online survey of the Abdominal and Gastrointestinal Imaging Study Group concerning the performance of abdominal radiological tests in the German-speaking countries generally show the surveyed radiologists to be mostly compliant with guidelines, with, however, there being to some extent heterogeneity in terms of acquired contrast phases and further technical examination details. The results should thus prompt the development and issuing of comprehensive and evidence-based specific recommendations for radiological imaging of the abdomen.

\section{Literatur}

1 Schreyer AG, Wessling J, Kinner S et al. Aktuelle Schwerpunkte und Literatur der Abdominalradiologie im deutschsprachigen Raum - Teil 1: Gastrointestinaltrakt. Fortschr Röntgenstr 2015, in press

2 Grenacher L, Juchems M, Holzapfel K et al. Aktuelle Schwerpunkte und Literatur der Abdominalradiologie im deutschsprachigen Raum - Teil 2: Parenchymatöse Oberbauchorgane. Fortschr Röntgenstr 2015, in press

3 Schleder S, Dendl LM, Pawlik M et al. MR enterography sequence evaluation for patients with Crohn's disease. Fortschr Röntgenstr 2013; 185: $440-445$

4 Silva AC, Evans JM, McCullough AE et al. MR imaging of hypervascular liver masses: a review of current techniques. Radiographics 2009; 29: $385-402$
5 Tirkes T, Menias CO, Sandrasegaran K. MR imaging techniques for pancreas. Radiol Clin North Am 2012; 50: 379-393

6 Yao XZ, Kuang T, Wu L et al. Comparison of diffusion-weighted MRI acquisition techniques for normal pancreas at 3.0 Tesla. Diagn Interv Radiol 2014; 20: 368 -373

7 Leitlinien. AdWMFA-SK. AWMF-Regelwerk „Leitlinien“. In. 2012; 1. Auflage ed

8 Leifeld L, Germer CT, Bohm S et al. S2k guidelines diverticular disease/ diverticulitis. Z Gastroenterol 2014; 52: 663-710

9 Schreyer AG, Layer G. S2k Guidlines for Diverticular Disease and Diverticulitis: Diagnosis, Classification, and Therapy for the Radiologist. Fortschr Röntgenstr 2015; DOI: 10.1055/s-0034-1399526

10 Field MJ, Lohr KN. Clinical practice guidlines: Direction for a new program. Wahington (DC): National Academic Press; 1990

11 Hugler S. AWMF: more than guideline development. Dtsch Med Wochenschr 2013; 138: 1926-1927

12 Kopp I, Muller W, Lorenz W. Guidelines in the AWMF system: position and perspectives. Z Arztl Fortbild Qualitatssich 2003; 97: 733-735

13 Seufferlein T, Porzner M, Becker T et al. S3-guideline exocrine pancreatic cancer. Z Gastroenterol 2013; 51: 1395 - 1440

14 Sommer CM, Stampfl U, Kauczor HU et al. National S3 guidelines on hepatocellular carcinoma. Radiologe 2014; 54: 642 - 653

15 Yokoe M, Takada T, Mayumi T et al. Japanese guidelines for the management of acute pancreatitis: Japanese Guidelines 2015. J Hepatobiliary Pancreat Sci 2015; 22: 405-432

16 Goldberg DT, Selina; Maly, Tim; Aldrich, Will. 2015, http://www.surveymonkey.com

17 Moehler M, Al-Batran SE, Andus T et al. German S3-guideline "Diagnosis and treatment of esophagogastric cancer". Z Gastroenterol 2011; 49: $461-531$

18 Erturk SM, Mortele KJ, Oliva MR et al. Depiction of normal gastrointestinal anatomy with MDCT: comparison of low- and high-attenuation oral contrast media. Eur J Radiol 2008; 66: 84-87

19 Gligorievski A. CT evaluation of gastric lymphoma. Prilozi 2009; 30: $125-138$

$20 \mathrm{Kim} \mathrm{HJ}$, Kim AY, Lee JH et al. Positioning during CT gastrography in patients with gastric cancer: the effect on gastric distension and lesion conspicuity. Korean J Radiol 2009; 10: 252-259

21 Huber W, Schmid RM. Diagnosis and treatment of acute pancreatitis. Current recommendations. Internist (Berl) 2011; 52: 823-830, 832

22 Tannapfel A, Wittekind C. The current TNM system for gastrointestinal tumors part II. Pathologe 2010; 31: 348-352

23 Barral M, Taouli B, Guiu B et al. Diffusion-weighted MR imaging of the pancreas: current status and recommendations. Radiology 2015; 274: $45-63$

24 Merkle EM, Zech CJ, Bartolozzi C et al. Consensus report from the 7th International Forum for Liver Magnetic Resonance Imaging. Eur Radiol 2015; DOI: 10.1007/s00330-015-3873-2

25 Song do S, Bae SH. Changes of guidelines diagnosing hepatocellular carcinoma during the last ten-year period. Clin Mol Hepatol 2012; 18: $258-267$

26 Chen N, Motosugi U, Morisaka H et al. Added Value of a Gadoxetic Acidenhanced Hepatocyte-phase Image to the LI-RADS System for Diagnosing Hepatocellular Carcinoma. Magn Reson Med Sci 2015; DOI: $10.2463 / \mathrm{mrms} .2014-0149$

27 Purysko AS, Remer EM, Coppa CP et al. LI-RADS: a case-based review of the new categorization of liver findings in patients with end-stage liver disease. Radiographics 2012; 32: 1977 - 1995

28 Alvaro D, Cannizzaro R, Labianca $R$ et al. Cholangiocarcinoma: A position paper by the Italian Society of Gastroenterology (SIGE), the Italian Association of Hospital Gastroenterology (AIGO), the Italian Association of Medical Oncology (AIOM) and the Italian Association of Oncological Radiotherapy (AIRO). Dig Liver Dis 2010; 42: 831 - 838

29 Dietrich CF, Cui XW, Boozari B et al. Contrast-enhanced ultrasound (CEUS) in the diagnostic algorithm of hepatocellular and cholangiocellular carcinoma, comments on the AASLD guidelines. Ultraschall in Med 2012; 33: S57-S66

30 Iavarone M, Piscaglia F, Vavassori S et al. Contrast enhanced CT-scan to diagnose intrahepatic cholangiocarcinoma in patients with cirrhosis. J Hepatol 2013; 58: 1188 - 1193

31 Rimola J, Forner A, Reig $M$ et al. Cholangiocarcinoma in cirrhosis: absence of contrast washout in delayed phases by magnetic resonance imaging avoids misdiagnosis of hepatocellular carcinoma. Hepatology 2009; 50: $791-798$ 
32 Wildner D, Bernatik T, Greis C et al. CEUS in hepatocellular carcinoma and intrahepatic cholangiocellular carcinoma in 320 patients - early or late washout matters: a subanalysis of the DEGUM multicenter trial. Ultraschall in Med 2015; 36: 132 - 139

33 Asayama Y, Yoshimitsu K, Irie $\mathrm{H}$ et al. Delayed-phase dynamic CT enhancement as a prognostic factor for mass-forming intrahepatic cholangiocarcinoma. Radiology 2006; 238: 150 - 155

34 Schreyer AG, Ludwig D, Koletzko S et al. Updated German S3-guideline regarding the diagnosis of Crohn's disease - implementation of radiological modalities. Fortschr Röntgenstr 2010; 182: 116-121

35 Stallmach A, Hoffmann J, Preiss JC. Updated S3 guideline on diagnosis and treatment of Crohn's disease: up to date or new? Z Gastroenterol 2014; 52: $1387-1388$

36 Pox C, Aretz S, Bischoff SC et al. S3-guideline colorectal cancer version 1.0. Z Gastroenterol 2013; 51: $753-854$

37 Neri E, Halligan S, Hellstrom $M$ et al. The second ESGAR consensus statement on CT colonography. Eur Radiol 2013; 23: 720 - 729
38 Spada C, Stoker J, Alarcon O et al. Clinical indications for computed tomographic colonography: European Society of Gastrointestinal Endoscopy (ESGE) and European Society of Gastrointestinal and Abdominal Radiology (ESGAR) Guideline. Endoscopy 2014; 46: 897-915

39 Grenacher L, Schwarz M, Lordick F et al. S3 guideline - diagnosis and treatment of gastric carcinoma: relevance for radiologic imaging. Fortschr Röntgenstr 2012; 184: 706 - 712

40 Schreyer AG, Jung $M$, Riemann JF et al. S3 guideline for chronic pancreatitis - diagnosis, classification and therapy for the radiologist. Fortschr Röntgenstr 2014; 186: 1002 - 1008

$41 \mathrm{Vogl} \mathrm{TJ}$, Al-Nawas B, Beutner D et al. Updated S2K AWMF guideline for the diagnosis and follow-up of obstructive sialadenitis-relevance for radiologic imaging. Fortschr Röntgenstr 2014; 186: 843-846

42 Vogl TJ, Schmiegel W, Pox C et al. S3 guideline - Diagnosis and treatment of colorectal carcinoma: relevance for radiologic imaging and interventions. Fortschr Röntgenstr 2013; 185: 699-708 